Jérôme Courduriès, 2014, « Expérience homosexuelle et parenté. Des relations familiales contrastées », Dialogue, Dossier Homosexualités et familles, 203, pp. 77-88. Version auteur

\title{
Expérience homosexuelle et parenté Des relations familiales contrastées
}

\author{
Jérôme Courduriès
}

\section{Résumé}

Cet article examine, du point de vue de l'anthropologie, la nature des relations que les personnes homosexuelles entretiennent aujourd'hui avec les membres de leur parenté, point encore assez obscur des travaux en sciences sociales menés sur l'homosexualité. Il s'appuie sur des enquêtes menées entre 2000 et 2011 auprès de cinquante-neuf hommes gays en couple et de jeunes adultes gays et lesbiennes en rupture familiale. Les données révèlent que, une fois levé le secret longtemps imposé sur leur orientation sexuelle, le plus souvent les relations des hommes en couple se redéploient avec leurs apparentés. Dans le même temps, des jeunes homosexuel(le)s souffrent encore de l'exclusion familiale du fait de leur orientation sexuelle. Illustré de quelques cas, l'article montre que les relations entretenues par les homosexuel(le)s avec leurs apparentés sont en réalité très contrastées.

\section{Mot-clés}

homosexualité, famille, parents, relation, secret, rupture.

Les travaux publiés ces dernières années dans la littérature internationale en sciences sociales suggèrent des changements de grande ampleur dans la façon de vivre l'homosexualité et du point de vue de la perception sociale des mœurs amoureuses et sexuelles. Néanmoins, ces changements ne s'opèrent pas rapidement ni partout. En effet, à y regarder de plus près, l'expérience des hommes et femmes homosexuel(le)s apparaît très contrastée.

Plusieurs travaux en sciences sociales portant sur la «carrière homosexuelle », au sens de l'École de Chicago, ont montré que les homosexuel(le)s, lorsqu'ils ou elles prenaient conscience de leur orientation sexuelle et entraient véritablement dans l'homosexualité, s'éloignaient irrémédiablement de leur famille, en particulier de leurs parents, et tissaient de nouvelles relations avec des ami(e)s partageant la même orientation sexuelle et construisaient ainsi de véritables familles d'élection (Weston, 1991). Cela correspond certainement encore aujourd'hui à l'expérience biographique de certaines personnes homosexuelles. Néanmoins, de nombreux travaux montrent que la réalité est beaucoup plus nuancée. Les liens avec la parenté peuvent certes se rompre durablement; j'illustrerai ces ruptures à partir d'une enquête ethnographique menée en 2010 et 2011 auprès de jeunes en rupture familiale du fait de leur homosexualité. Souvent, cependant, après s'être distendus au moment du coming out, les liens avec les apparentés se resserrent et les personnes homosexuelles, éventuellement en couple et parents, s'inscrivent dans un réseau de parenté large. Les données que j'ai recueillies dans les années 2000 à 2010 à l'occasion de mes recherches sur la conjugalité homosexuelle masculine, ainsi que d'autres travaux menés en France et ailleurs, nous permettront de réfléchir à la manière dont les personnes homosexuelles s'inscrivent aussi dans des relations familiales consanguines et alliées. Compte tenu du cadre contraint de l'article, je présenterai seulement quelques cas qui me semblent particulièrement emblématiques des témoignages recueillis.

\section{Être homosexuel(le) : du secret au dévoilement}

Qu'il s'agisse de situations de rejet réellement vécues ou de la peur éprouvée d'être rejeté, il subsiste des situations où l'orientation homosexuelle reste de nos jours un stigmate porteur de 
rejet familial (Goffman, 1996). La quasi-absence, en particulier durant leurs jeunes années, de «modèles » homosexuels positifs dans l'environnement culturel et la dévalorisation de l'homosexualité, toujours vive dans certains milieux, expliquent que, parmi les personnes que j'ai interviewées, nombreuses sont celles qui ont un temps dû tenir leur famille à distance.

Tous les hommes interrogés ont évoqué la peur qu'ils ont longtemps ressentie de se voir rejetés par leur entourage, en particulier leurs parents. Les raisons qui les ont poussés à faire leur coming out tiennent à leur difficulté à vivre ce qu'ils qualifient eux-mêmes de « double vie ». Les mots qu'ils utilisent sont évocateurs de la dissimulation : «tromper », « cacher », « secret ». Ceux employés à l'occasion du récit de leur coming out ne suggèrent pas moins le climat lourd d'un secret longtemps gardé : « avouer », « révéler », « dévoiler ».

Le secret se fait d'autant plus impérieux que la personne homosexuelle peut entendre ses proches plaisanter aux dépens des personnes homosexuelles et même parfois exprimer des propos homophobes. Lorsque mes interlocuteurs, hommes en couple gay, font part de leurs craintes concernant les réactions familiales, ils pensent particulièrement à celle de leur propre père. La peur de la réaction paternelle s'explique en grande partie par le fait que le père est généralement perçu comme celui qui formule les attentes les plus traditionnelles à l'égard de son fils. En juin 1994, Vincent passe son bac. C'est le moment où il commence son journal, qui se termine en décembre 2000 au terme de deux cent quarante feuillets ${ }^{1}$. Il écrit, avoir remarqué chez son père un " mépris pour les homosexuels »: «Il ne les conçoit que comme des pédales, des filles inavouées et complexées et se demande, pour un couple lesbien particulièrement, qui fait l'homme et qui fait la femme. C'est tellement méprisant? une telle remarque, tellement blessant, tellement intolérant » (Journal, samedi 12 décembre 1998, p.72).

Qui fait l'homme, qui fait la femme ? La «sempiternelle question », entendue au mieux dans les blagues de potache, au pire dans un contexte plus violent, rapportée également par Line Chamberland et Christelle Lebreton (2008, p. 148) et dans ma propre recherche doctorale (Courduriès, 2006), témoigne de la confusion dans le sens commun entre orientation sexuelle et identité de genre : parce qu'on est homosexuel(le) on ne serait pas vraiment homme (femme)'.

Parmi les moments favorables au dévoilement de soi, la contractualisation d'un Pacs, en particulier lorsqu'elle s'accompagne d'une forme de ritualisation inspirée des pratiques matrimoniales, est l'occasion de réunir autour du couple l'ensemble des proches et impose que ce qui était jusque-là passé sous silence soit montré. Il semble que lorsqu'elle est associée à la conjugalité l'homosexualité devient moins problématique.

\section{Les effets de l'entrée en conjugalité sur la visibilité familiale de l'homosexualité}

Dans notre société, la vie conjugale et familiale n'est plus organisée par sa famille d'origine. Néanmoins il reste important pour nombre de personnes que leur couple soit intégré dans le tissu des relations de parenté. Mais cette quête, à la différence des couples hétérosexuels, nécessite pour les personnes homosexuelles que soit explicitée, avec leurs parents, leur orientation sexuelle: il ne s'agit pas simplement d'accueillir le conjoint ou la conjointe choisi(e) par son enfant, mais d'accepter l'homosexualité de son fils ou de sa fille. Le fait de

$1 \quad$ Ce journal est déposé à l'Association pour l'autobiographie.

Jérôme Courduriès, anthropologue, maître de conférences, Université Toulouse 2 le Mirail, laboratoire LISST-

CAS (UMR 5193) ; jerome.courduries@univ-tlse2.fr 
vivre en couple a un effet révélateur. À l'inverse, la difficulté à dire son homosexualité à ses proches pèse sur l'entrée dans la conjugalité et dans la corésidence. Par ailleurs, la relative banalisation de l'homosexualité dans notre société a favorisé l'engagement des personnes homosexuelles dans la conjugalité et, dans le même temps, la vie de couple des individus peut jouer un rôle déterminant dans l'acceptation par l'entourage de leur homosexualité (Schiltz, 1997; Adam, 1999). Pour le dire autrement, la conjugalité est plus acceptable que le «multipartenariat» supposé et a sur l'homosexualité un effet normalisant; c'est particulièrement vrai pour un certain nombre d'homosexuel(le)s qui ont fortement intégré le stigmate lié à leur orientation sexuelle (Costechareire, 2014). Il existe donc une nette corrélation entre adoption d'un mode de vie conjugal et connaissance familiale de l'homosexualité. S'agit-il d'une relation de cause à effet et dans quel sens opère-t-elle ? La corésidence conjugale est-elle rendue possible par l'information préalable de la famille ou bien est-ce l'inverse ? L'une et l'autre de ces logiques sont opérantes et se retrouvent tout à tour mobilisées dans les histoires que j'ai recueillies.

Dès que ses parents ont tous les deux su qu'il était en couple, Simon (28 ans, chef de projet) a régulièrement emmené son compagnon, Bruno (32 ans, cadre), dans sa famille pour un weekend ou quelques jours. Dès le premier soir, la mère de Simon avait préparé l'ancienne chambre de son fils et c'est « tout naturellement », dit-il, qu'ils ont dormi dans le même lit. Avec le temps, Simon dit que Bruno est devenu le «quatrième enfant» de sa mère. William (29 ans, vendeur) dit, lui aussi, que Florent (34 ans, chef de chantier) est aujourd'hui « comme le troisième fils » de sa mère. Du point de vue de Simon et de William, leur intégration et celle de leur compagnon dans leur famille d'origine sont réussies - jusqu'à devenir des alliés, au sens de l'anthropologie de la parenté.

Les mariages sont souvent l'occasion de réunir les familles, parfois dispersées géographiquement. Ce fut le cas lorsque le frère aîné de Simon s'est marié avec sa fiancée ; proches et moins proches ont tous été invités et Bruno l'a été aussi. C'est véritablement à cette occasion que Simon a compris, avec émotion, que son compagnon avait été intégré par sa famille dans le premier cercle des proches, qu'il était, pour lui-même et les autres membres de sa parenté, un allié parmi d'autres. Il cite, pour illustrer ce sentiment, un moment qui lui semble révélateur, lorsque le photographe immortalise la famille unie autour des jeunes mariés : «Pendant la séance photo, ma mère a dit : "je vous veux tous les 6 - mon grand frère avec sa femme, mon petit frère avec sa copine et moi avec Bruno !»

Cependant, visibilité et intégration familiale ne signifient pas toujours que l'orientation sexuelle a été verbalisée, discutée en famille. Aujourd'hui, Jérémy (31 ans, aide-soignant) et Jean (41 ans, chef d'entreprise) sont invités régulièrement chez les parents de Jérémy et participent aux différents événements familiaux. Pourtant, leur relation n'a jamais été véritablement verbalisée : «Ma famille m’a jamais demandé [...] : "Est-ce que c'est ton copain, est-ce que c'est ton mec ?" Jamais, jamais. On invite Jérémy et Jean, quoi. Voilà. » Ce couple ne constitue pas un cas isolé puisque sur la totalité des cinquante-neuf gays rencontrés dans le cadre de mes enquêtes doctorale et post-doctorale, onze rapportent une expérience semblable: les couples fréquentent leurs familles respectives, échangent avec elles des invitations et des cadeaux, à l'occasion de Noël et des anniversaires ; pourtant le compagnon n'a jamais été ouvertement présenté comme tel.

Cependant, le chemin vers une intégration complète du couple dans la vie familiale n'est pas toujours linéaire. Certains moments de la vie de la parenté donnent l'occasion de nouveaux accrocs. Fabien (39 ans, directeur commercial) raconte le mariage de sa sœur cadette. Son 
compagnon s'était beaucoup investi auprès de la future mariée pour la préparation de ce mariage. Il fut remercié au microphone par le père de Fabien, devant tous les convives ; une reconnaissance publique qui donna toute leur place à Fabien et son compagnon dans sa parenté. Pourtant, Fabien avait dû se battre pour imposer sa relation amoureuse. Dans un premier temps, ils n'avaient en effet pas été invités au déjeuner des fiançailles qui réunissait les deux familles. Alors que Fabien et son compagnon fréquentaient souvent la maison familiale, et, qu'en apparence, leur présence ne posait problème à personne, son père et sa sœur, craignant la réaction de la belle-famille de la future épouse, lui avaient fait comprendre que lui seul était le bienvenu. La famille traversa alors une crise : son frère menaça de ne pas honorer l'invitation, sa sœur aînée "fit un scandale » et la future mariée fondit en larmes. Mais au moment où Fabien me confie son témoignage, tout cela lui semble bien loin. Avec Gérald, son compagnon d'aujourd'hui, ils sont de toutes les fêtes familiales.

Aucune enquête ethnographique n'a encore été menée en France auprès de couples de même sexe ayant contracté un mariage. Il serait en particulier intéressant de savoir s'ils reprennent à leur compte les rituels matrimoniaux mis en œuvre par leurs homologues hétérosexuels, s'ils organisent une fête pour célébrer leur union et, dans ce cas, s'ils invitent familles et amis. Cependant, d'autres travaux se sont intéressés à la manière dont les couples de même sexe contractant un pacte civil de solidarité, dans une période où le mariage ne leur était pas encore ouvert, montrent comment la famille pouvait y être associée (Rault, 2009 ; Courduriès, 2011). L'analyse de Wilfried Rault montre par exemple que, lorsqu'une fête est organisée, la famille occupe souvent une place seconde par rapport au cercle amical. Il voit là le résultat d'une difficile acceptation familiale de l'homosexualité et « une plus grande latitude que peuvent parfois avoir les gays et lesbiennes vis-à-vis de leur réseau de parenté » (Rault, 2014). Il faut dire également, comme le signale le sociologue, que le statut même du Pacs, qui n'est pas un mariage, permettait aux contractants de ne pas se sentir systématiquement obligés d'associer étroitement leur famille.

Malgré les enseignements de mes recherches sur la conjugalité homosexuelle, il ne faisait aucun doute que les discriminations, les discours haineux et les violences physiques à l'encontre de personnes homosexuelles rapportés par les médias devaient aussi avoir quelquefois des échos dans la sphère familiale. L'existence, en France, de dispositifs associatifs de remédiation destinés à de jeunes homosexuel(le)s en situation de rupture familiale en est une illustration. Mais à ce jour, malgré certains discours sociaux dénonçant l'homophobie, on connait peu de choses sur cette expérience de la remise en cause du lien parent-enfant, pourtant socialement présenté comme inaltérable.

\section{Jeunesse, homosexualité et rejet familial}

De grands changements semblent être intervenus en Europe occidentale dans la manière dont les jeunes homosexuel(le)s vivent la découverte de leur homosexualité. À partir d'une enquête menée en Espagne, l'anthropologue José Ignacio Pichardo Galán » (2010, p. 384) note en effet que, contrairement à leurs aînés, les adolescent(e)s homosexuel(le)s d'aujourd'hui « parlent de sexe sans détour ». Ces résultats sont certainement à nuancer selon les contextes ; on peut néanmoins s'accorder sur le fait que l'homosexualité ne constitue plus systématiquement un tabou chez les jeunes. Cependant, le tabou pèse encore dans de nombreuses situations, tout particulièrement au sein de la famille. Et lorsqu'il survient, le rejet du (de la) jeune homosexuel(le) par sa famille pèse sur son bien-être émotionnel et affectif, jusqu'à lui faire ressentir parfois un sentiment de honte (Courduriès, 2014), comme le souligne le document édité après une enquête par questionnaire complétée sur internet par 
près de cinq cents répondants des deux sexes (Dorais, Chollet, 2012, p. 57). Il a aussi des conséquences matérielles directes et importantes, en particulier, bien sûr, lorsque les jeunes sont financièrement dépendants.

Yohann (19 ans, sans emploi) vivait avec son père (vendeur) et sa mère (sans emploi). Un jour, son père a surpris des échanges sans équivoque que Yohann avait avec un garçon rencontré sur internet. La scène fut d'une grande violence. Le jeune homme explique que son père « a pété un plomb » : «Il s'est mis à me crier dessus, m'a soulevé de la chaise et m'a poussé. Je suis tombé et il continuait à crier. [...] j'étais flippé, t'imagines même pas. Il me traitait de pédé, qu'il l'avait toujours su. Il disait n'importe quoi... Il mélangeait tout : que j'avais pas voulu faire du foot, que j'avais trop de copines. Il s'en est pris à ma mère. Il était fou. " Finalement, le père de Yohann a enfoncé son poing dans la porte et est sorti de la chambre, excédé. Poussé par la crainte que lui inspirait son père et la certitude que la situation familiale était irréversible, dès que l'association qu'il avait contactée par téléphone l'a informé qu'un appartement l'attendait, Yohann est parti un après-midi avec un sac de sport rempli de vêtements. Sa sœur, de quelques années son aînée et dont il était très proche, en pleurs, lui a donné cinquante euros ("C'était beaucoup d'argent pour nous »). Au moment où Yohann et moi discutons, sa sœur et lui se téléphonent régulièrement mais à leur mère qui demande où est Yohann, sa sœur a seulement répondu qu'il était hébergé par une association, sans préciser laquelle, ni où.

Les parents ne sont pas les seules personnes à jouer un rôle dans la biographie des jeunes homosexuel(le)s que j'ai rencontré(e)s. Les frères et sœurs en particulier occupent une place centrale dans de nombreux récits.

\section{Les germains, tantôt des séides des parents homophobes, tantôt des alliés}

Les anthropologues américains Elaine Cumming et David Schneider, à partir d'une enquête menée à Kansas City, envisagent le lien entre frères et sœurs à l'âge adulte comme un lien particulièrement fort et solidaire ${ }^{2}$. Cela ne veut pas dire qu'ils sont tous très unis, mais que les liens de solidarité les plus intenses dans la parenté à l'âge adulte s'observeraient parmi les frères et sœurs et s'étendraient même aux cousins issus de germains (Cumming, Schneider, 1961, p. 501). L'anthropologue Agnès Martial le confirme à partir de ses enquêtes auprès des enfants élevés dans un foyer recomposé et n'ayant entre eux aucun lien de sang (Martial, 2003). Mais force est de constater qu'un certain nombre d'événements peuvent troubler les relations familiales et fragiliser, dans des contextes sans doute spécifiques, un lien fraternel pourtant perçu le plus souvent à l'époque contemporaine comme « indéfectible » (Fine, 2007, p. 71).

Il est très difficile de connaître le rôle qu'ont choisi d'endosser les frères et sœurs de personnes homosexuelles lorsque leur orientation sexuelle a été dévoilée et d'en faire une typologie. Car les enquêtes empiriques manquent. Dans ma propre recherche sur la conjugalité gay, la grande majorité des hommes que j'ai rencontrés ont présenté leurs frères et sœurs comme des complices, des alliés, pouvant parfois s'engager explicitement en leur faveur auprès de leurs parents (Courduriès, 2011, p. 133).

Le récit de Lucie (22 ans, sans emploi) et de Carine (21 ans, sans emploi) amène pourtant à s'interroger sur la réaction des frères et sœurs face à la découverte de l'homosexualité de leurs

2 L'article d'Elaine Cumming et de David Schneider est cité dans Crenner, Déchaux, Herpin, 2000. Jérôme Courduriès, anthropologue, maître de conférences, Université Toulouse 2 le Mirail, laboratoire LISSTCAS (UMR 5193); jerome.courduries@univ-tlse2.fr 
germains. Le frère de Lucie, de quatre ans son cadet, a révélé l'homosexualité de sa sour à leurs parents. Celui de Carine, quant à lui, a emboîté le pas à son beau-père qui parlait de déshonneur, de honte ; tous les deux ont été jusqu'à enfermer Carine et menacer Lucie. Les frères apparaissent ici comme des suppôts des parents. Carine et Lucie ont réussi à se rendre dans une association homosexuelle de leur département. Elles n'avaient alors aucun plan véritable, mais elles étaient sûres d'une chose: elles ne pouvaient envisager un avenir commun près de leurs familles.

Christelle Hamel (2003) a mené une recherche sur les relations amoureuses, la sexualité et la gestion des risques d'infection par le VIH, auprès de soixante-dix jeunes femmes et hommes d'origine maghrébine appartenant à des familles modestes, dont les parents appartenaient au monde ouvrier; parmi ces jeunes femmes six étaient lesbiennes. Christelle Hamel (2012, p. 102) explique que les frères se substituent souvent au père et surveillent les faits et gestes de leurs sœurs dans le but de veiller à leur bonne moralité, voire de les « ramener dans le droit chemin ». On retrouve ce rôle d'entrepreneur de morale (Becker, 1963) non seulement dans les grandes cités périurbaines, mais aussi parfois en milieu rural, comme le montrent les enquêtes menées par Isabelle Clair (2008).

Parmi les jeunes que j'ai rencontrés, tous n'ont pas été abandonnés par leurs germains. Quelques-uns ont même conservé des relations avec eux et, malgré l'éloignement géographique, leur donnent des nouvelles et parfois les rencontrent. Si le lien fraternel dans notre société est second par rapport au lien de filiation (Crenner, Déchaux, Herpin, 2000, p. 218), nous voyons que dans certains cas, où la rupture entre une personne homosexuelle et ses propres parents semble consommée, les liens entre germains sont ceux qui restent et par lesquels subsiste une relation même ténue avec la parenté consanguine. Outre les frères et sœurs qui, le plus souvent, sont les premiers confidents et font preuve de solidarité en cas de conflits, existe-t-il d'autres membres de la parenté avec lesquels ces jeunes gardent des liens ?

\section{Liens avec les autres membres de la parenté}

Des recherches menées dans des milieux populaires au Brésil et en Espagne donnent à voir des familles où l'homosexualité de l'un des membres ne vient pas altérer les liens de solidarité intrafamiliale. Peut-être même au contraire. José Ignacio Pichardo Galán (2014) montre que dans les familles espagnoles de son corpus, dans un contexte de crise économique, les personnes homosexuelles et, le cas échéant, leur conjoint(e), sont invités à remplir le rôle qui leur est dévolu en se montrant solidaires, y compris financièrement, de leurs aînés. Flávio Luiz Tarnovski évoque pour sa part les situations où des hommes homosexuels, qu'ils soient engagés ou non dans une relation sentimentale, assument les obligations qui leur sont échues par la coutume envers leurs neveux et nièces, en cas de défaillance ou d'absence de leurs parents (2014).

Je n'ai observé aucune forme de solidarité aussi poussée dans mes propres recherches en France. Néanmoins, certains apparentés semblent avoir une place tout à fait privilégiée dans la parentèle de mes enquêté(e)s. Ainsi, Pauline (23 ans, sans emploi) dit conserver surtout des relations avec une tante paternelle, sa marraine : « Ma tante, c'est un peu comme ma maman. [...] En plus on se ressemble. Je ne ressemble pas du tout à ma mère, je ressemble à ma tante. Et du coup, voilà, on s'entend merveilleusement bien. Puis elle est pas très loin. » $\mathrm{Ce}$ « On se ressemble » ne va pas sans faire écho aux travaux d'Agnès Fine sur le parrainage (1994). Ses enquêtées se trouvaient aussi quelques ressemblances avec leur marraine, exprimant ainsi la force d'une croyance très ancienne établissant une sorte d'hérédité spirituelle entre 
parrain/marraine et filleul(e). Manifestement, Pauline se sent plus fille de sa tante que de sa mère : "J'ai des relations très compliquées avec ma mère. En fait j'ai plus d'affection et d'attention de ma tante que de ma mère. [...] c'est vrai qu'elle est tout ce que je voudrais que ma mère soit. Elle est attentionnée. Elle fait super attention à moi. Dès qu'elle voit que ça ne va pas, elle me demande ce que j'ai. » Sans minimiser l'importance des centres d'intérêt que Pauline dit avoir en commun avec sa tante ni les égards que sa tante a pu lui manifester de longue date, un autre élément caractérise la relation particulière que toutes les deux entretiennent encore aujourd'hui, même à distance. Sa tante connaît son homosexualité depuis que Pauline a 16-17 ans (ce fut d'ailleurs la première dans sa famille) et ne semble y trouver aucun motif de contrariété.

\section{Conclusion}

L'expérience vécue par les femmes et les hommes homosexuels, du point de vue de leurs relations de parenté, est, depuis les années 2000, autrement plus contrastée qu'on ne pouvait le penser. Vivre des relations amoureuses et sexuelles avec des personnes du même sexe, être gay ou lesbienne n'implique pas nécessairement de rompre avec toute sa parenté (Fine, Courduriès, 2014). Les changements législatifs intervenus au cours des quinze dernières années et les débats qui les ont accompagnés n'y sont certainement pas étrangers. En outre, au-delà de l'occasion qu'elle représente de donner à voir ce qui était jusque-là passé sous silence, il est clair que l'entrée en conjugalité facilite l'acceptation familiale. On n'est plus seulement gay ou lesbienne ; on est également en couple. Qu'il ou elle soit concubin(e), partenaire, époux ou épouse, le compagnon, la compagne entre dans la parenté en tant qu'allié. Et lorsqu'un homme gay ou une femme lesbienne devient parent, il (elle) trouve une place nouvelle dans sa parenté consanguine (Herbrand, 2014; Gross, 2014): enfant de, il (elle) devient aussi ascendant.

Cependant, même en couple ou parents, tous ne parviennent pas à se faire accepter par leur famille. Les parcours des jeunes décrits plus haut, victimes de l'homophobie familiale, parfois même menacés dans leur intégrité physique par leurs propres père et mère, frère et sœur, nous rappellent singulièrement que nous ne sommes pas encore entrés dans une période où l'orientation homosexuelle serait considérée en tous lieux comme strictement équivalente à l'orientation hétérosexuelle.

\section{Bibliographie}

Adam, P. 1999. « Bonheur dans le ghetto ou bonheur domestique ? ", Actes de la recherche en sciences sociales, 128, 56-67.

Becker, H. S. 1963. Ousiders. Études de sociologie de la déviance, Paris, Métailié, 1985.

Chamberland, L.; Lebreton, C. 2008. "L'homosexualité au bureau. À l'heure des transformations du droit conjugal et familial canadien », dans V. Descoutures, M. Digoix, É. Fassin, W. Rault (sous la direction de), Mariages et homosexualités dans le monde. L'arrangement des normes familiales, Paris, Autrement, 139-150.

Clair, I. 2008. Les jeunes et l'amour dans les cités, Paris, Armand Colin, Collection « Individu et Société ».

Costechareire, C. 2014. «Le vécu de l'homosexualité féminine au regard de la tolérance et de 1'hostilité familiale », dans J. Courduriès et A. Fine (sous la direction de), Homosexualité et parenté, Paris, Armand Colin. 
Courduriès, J. 2006. "Les couples gays et la norme d'égalité conjugale », Ethnologie française, vol. 36, 4, 705-711.

Courduriès, J. 2011. Etre en couple (gay). Conjugalité et homosexualité masculine en France, Lyon, PUL.

Courduriès, J. 2014. «Rompre avec sa famille. Jeunesse, entrée dans l'homosexualité et rejet familial », dans J. Courduriès et A. Fine (sous la direction de), Homosexualité et parenté, Paris, Armand Colin.

Crenner, E. ; Déchaux, J.-H. ; Herpin, N. 2000. «Le lien de germanité à l'âge adulte. Une approche par l'étude des fréquentations », Revue française de sociologie, 41, 2, 211-239.

Cumming, E. ; Schneider, D. M. 1961. « Sibling solidarity : a property of American kinship », American anthropologist, 63, 3, 498-507.

Dorais, M. ; Chollet, I. 2012. Etre homo aujourd'hui en France, Le Triadou, H\&O.

Fine, A. 2007. "Les fratries en Europe. Aperçu sur quelques orientations de recherche en anthropologie ", dans M. Oris, G. Brunet, E. Widmer, A. Bideau (sous la direction de), Les fratries. Une démographie sociale de la germanité, Bern, Peter Lang, 47-78.

Fine, A. 1994, Parrains, marraines. La parenté spirituelle en Europe, Paris : Fayard.

Fine, A. ; Courduriès, J. 2014. « Homosexualité et parenté. Introduction », dans Homosexualité et parenté, Paris, Armand Colin.

Goffman, E. 1996 (1963). Stigmate. Les usages sociaux des handicaps, Paris, Minuit.

Gross, M. 2014. " Grands-parents, homopaternité et gestation pour autrui en France », dans J.

Courduriès et A. Fine (sous la direction de), Homosexualité et parenté, Paris, Armand Colin.

Hamel, C. 2003. "L'intrication des rapports sociaux de sexe, de "race », d'âge et de classe : les effets sur la gestion des risques d'infection par le VIH chez les français descendants des migrants du Maghreb », Thèse d'anthropologie sociale et ethnologie, Paris, EHESS.

Hamel, C. 2012. " Devenir lesbienne : le parcours de jeunes femmes d'origine maghrébine », AGORA Débats /Jeunesse, 60, 93-105.

Herbrand, C. 2014. « Les rendre grands-parents. L'enjeu des relations intergénérationnelles au sein des coparentalités gaies et lesbiennes en Belgique », dans J. Courduriès et A. Fine (sous la direction de), Homosexualité et parenté, Paris, Armand Colin.

Martial, A. 2003. S'apparenter. Ethnologie des liens de familles recomposées, Paris, MSH.

Pichardo Galán, J. I. 2009. Entender la diversidad familiar. Relaciones homosexuales y nuevos modelos de familia, Barcelone, Bellaterra.

Pichardo Galán, J. I. 2010. "Les adolescents LGBT d'aujourd'hui », dans V. Blanchard, R. Pichardo Galán, J. I. 2014. "Maintenir les liens : diversité sexuelle et famille d'origine en Espagne ", dans J. Courduriès et A. Fine (sous la direction de), Homosexualité et parenté, Paris, Armand Colin.

Revenin et J.-J. Yvorel, Les jeunes et la sexualité : initiations, interdits, identités (XIX ${ }^{e}-X X I^{e}$ siècle), Paris, Autrement, 375-383.

Rault, W. 2009. L'invention du Pacs. Pratiques et symboliques d'une nouvelle forme d'union, Paris, Presses de Sciences Po.

Rault, W. 2014. «Des relations familiales à l'épreuve du Pacs », dans J. Courduriès et A. Fine (sous la direction de), Homosexualité et parenté, Paris, Armand Colin.

Schiltz, M.-A. 1997. «Parcours de jeunes homosexuels dans le contexte du VIH : la conquête de modes de vie », Population, 52, 6, 1485-1537.

Tarnovski, F. L. 2014. "Homosexualité et solidarité familiale : le cas des oncles gays au Brésil », dans J. Courduriès et A. Fine (sous la direction de), Homosexualité et parenté, Paris, Armand Colin.

Weston, K. 1991. Families we choose. Lesbians, Gays, Kinship, New York, Columbia University Press. 
Jérôme Courduriès, 2014, « Expérience homosexuelle et parenté. Des relations familiales contrastées », Dialogue, Dossier Homosexualités et familles, 203, pp. 77-88. Version auteur

\title{
Homosexual experience and family relationships Contrasted family relations
}

\begin{abstract}
Starting out from an anthropological perspective, this article examines the nature of the relations that homosexual people maintain nowadays with members of their family. This is an issue that still remains shrouded in relative obscurity in works in the social sciences devoted to homosexuality. It looks at the results of surveys conducted between 2000 and 2011 with fifty-nine gay men living in couples and young gay adults and lesbians having become separated from their families. The data reveal that, once the secret that had so long been imposed on their sexual orientation has come out, most often the relations of men living in couples become redeployed with the members of their family. At the same time, young homosexuals still suffer from family exclusion due to their sexual orientation. The article takes a few examples and goes on to show that the relations maintained by homosexuals with their family members are in reality extremely contrasted.
\end{abstract}

\section{Keywords}

Homosexual, family, parents, relation, secret, family separation. 